\title{
EPR Spectroscopy of Interdoublet Transitions in High-Spin Iron: Applications to Transferrin Oxalate
}

\author{
Kutbuddin S. Doctor, B. J. Gaffney," Gabriel Alvarez, ${ }^{\dagger}$ and Harris J. Silverstone \\ Department of Chemistry. The Johns Hopkins University, Baltimore, Maryland 21218-2685
}

Received: December 4, 1992; In Final Form: January 19, 1993

\begin{abstract}
With an interdoublet transition (a transition between two states belonging to different Kramer doublets) as a unifying example, we discuss the contribution of individual resonances to the EPR spectrum of high-spin iron via two kinds of graphical methods that correlate resonance magnetic field with orientational angles and transition probabilities: a "density dot plot" and a multidimensional "spherical colored-dot plot". We reexamine two aspects of the spectra of the oxalate complex of diferric transferrin: the role of the $2 \rightarrow 3$ interdoublet transition, including special line-shape considerations, and whether accurate values of $D$ and $E$ for the oxalate complex can be obtained from the EPR spectra alone.
\end{abstract}

\section{Introduction}

Heme proteins provided the first opportunities to compare the theory of EPR spectroscopy of high-spin iron in proteins with experiments. ${ }^{1-3}$ More recently, many proteins containing highspin, non-heme iron have been identified and have also become the subject of spectroscopic studies. $4-6$ At the same time, advances in computer hardware and software have facilitated more detailed comparisons of experimental and theoretical spectra than were possible at the time the theory was developed. In this paper, we take a new look at the theory of electron paramagnetic resonance (EPR) spectroscopy of high-spin ferric iron for cases where the Zeeman and spin-spin interaction terms in the Hamiltonian are of similar magnitudes. ${ }^{7}$ Since all six energy levels are closely spaced, in this case, transitions between non-Kramers pairs of levels (interdoublet transitions) are theoretically possible. To aid in understanding how all transitions from molecules at all orientations contribute to the composite spectrum, graphical methods are presented (including the use of Mathematica) to display the angular variation of resonant fields together with corresponding transition moments. The EPR spectra of transferrin oxalate at $\mathbf{X}$-band are discussed as an application of this graphical information. The conditions under which interdoublet transitions might be observed are evaluated.

The spin Hamiltonian for high-spin iron is

$$
H_{S}=\beta \mathrm{B} \cdot \mathrm{g} \cdot \mathrm{S}+\mathrm{S} \cdot \mathrm{D} \cdot \mathrm{S}+\text { (terms fourth order in spin) }
$$

Usually, it is only necessary to consider the terms second order in spin. In this case, with appropriate choice of axes, the EPRobservable fine structure terms in the spin Hamiltonian involve only two nonzero parameters, the tetragonal splitting constant $D$ and the rhombic splitting constant $E$, and the spin Hamiltonian reduces to

$$
H_{S}=g \beta \mathrm{B} \cdot \mathrm{S}+D\left(S_{z}{ }^{2}-{ }^{1} /{ }_{3} \mathrm{~S}^{2}\right)+E\left(S_{x}{ }^{2}-S_{y}{ }^{2}\right)
$$

For the case under discussion here, $g \beta B \cdot S \sim D$, calculation of the energies requires diagonalization of the Hamiltonian matrix. The complexities of the variation in resonant fields with angular orientation and with $D$ and $E$ have long been appreciated. Aasa ${ }^{7}$ summarized results for symmetries from axial $(E=0)$ to rhombic $(E / D=1 / 3)$ at selected orientations of molecular axes in the magnetic field. His summaries were given as plots of $D / h \nu$ vs $B / h \nu$, and his calculations were applied to predicting the turning points for the spectra of transferrin carbonate.

† Permanent address: Departamento de Fisica Teorica, Facultad de Ciencias Fisicas, Universidad Complutense, 28040 Madrid, Spain.
We are concerned here with the presentation of information when $D, E$, and $\nu$ are fixed. The resonance fields $B$ depend on the angles $\theta$ and $\phi$ that $B$ makes with the molecular axes and on which pair of states is in resonance. For each resonance, there is an associated transition probability. There is also the conversion from frequency-swept to field-swept line shape. How this information is averaged into the experimental spectrum is not always transparent from the final spectrum. With an interdoublet transition as a unifying example, we discuss the contribution of individual resonances to the final spectrum via two kinds of plots. The first is a "density dot plot" that correlates resonance magnetic field with orientational angles and transition probabilities. The second uses "spherical colored-dot plots". The first method is easily carried out with standard graphing programs. The second, more complicated method can be implemented via Mathematica.

The EPR spectra of diferric transferrin complexed with several bidentate anions have recently been assigned as arising from superposition of transitions between more than one pair of energy levels. ${ }^{8}$ When the symmetry is intermediate between rhombic and axial, as is the case for the oxalate complex of transferrin, transitions between levels 1 and 2,2 and 3 , and 3 and 4 theoretically all have significant intensity. However, for the "interdoublet" transition between levels 2 and 3, resonance fields are highly variable with angle and with $D$. As a result, special consideration of line-shape functions and distributions in $D$ must be made to evaluate whether $2 \rightarrow 3$ transitions can in fact be observed experimentally.

\section{Calculations}

Calculation of Energy Levels and Transition Probabilities. In a recent review, ${ }^{4}$ we have outlined calculations of energies for the EPR of $S=5 / 2\left(\mathrm{Fe}^{3+}\right)$. The special cases of axial and rhombic symmetry, and $D \gg h \nu$, were treated in some detail. The more complex case of symmetry intermediate between rhombic and axial, and where $D \sim h v$, is at the focus of the present paper. We have chosen parameters used in the simulation of the experimental EPR spectra of transferrin oxalate as an illustration: $D=0.26$ $\mathrm{cm}^{-1}, E=0.026 \mathrm{~cm}^{-1}$, and $\nu=9.22 \mathrm{GHz}\left(h \nu=0.31 \mathrm{~cm}^{-1}\right)$. These parameters differ slightly from those used earlier. ${ }^{4}$ How they were chosen will be discussed later. For now we illustrate the angular dependence of the resonant field and transition probability. Note that we have two functions, resonant field and transition probability, to represent versus two variables, $\theta$ and $\phi$, so that the information problem is how to make a four-dimensional plot.

The calculation of the energy levels of the spin Hamiltonian (2) within the space spanned by the $S=5 / 2$ sextet is straightforward: diagonalization on a computer. A FORTRAN program 


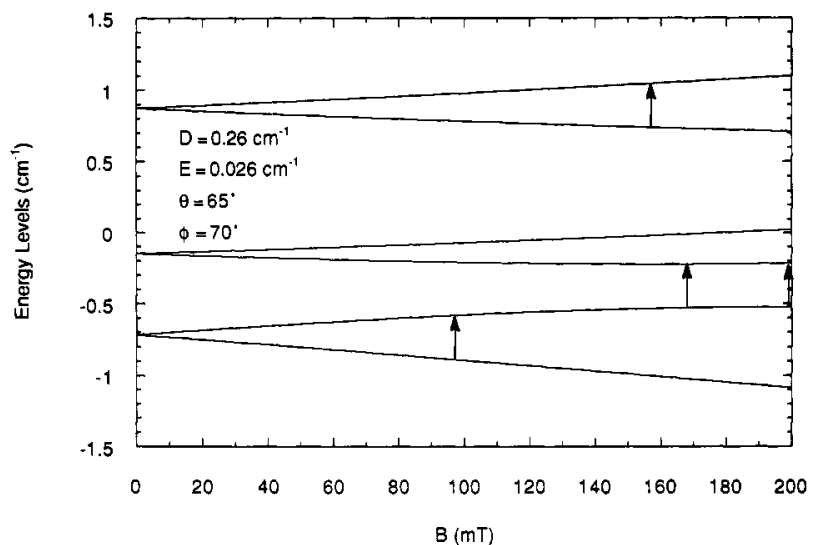

Figure 1. Energy levels for $S=5 / 2 \mathrm{Fe}^{3+}: D=0.26 \mathrm{~cm}^{-1}, E=0.026 \mathrm{~cm}^{-1}$, and orientation of $B$ is $(\theta, \phi)=\left(65^{\circ}, 70^{\circ}\right)$. The four arrows indicate the four instances at which the energy separations match the microwave frequency $\nu=9.22 \mathrm{GHz}: 97 \mathrm{mT}$ for the lowest Kramers doublet, 157 $\mathrm{mT}$ for the highest. There are $2 \rightarrow 3$ interdoublet transitions at 168 and $199 \mathrm{mT}$.

was described in ref 4 to take as input values of $D, E$, and $\nu$ and to give as output a table of the resonance fields $B$ and the squaredtransition-dipole matrix elements at a grid of values of $\theta$ and $\phi$ for each of the 15 possible transitions, $i j$, where $i$ runs from 1 to 5 , while $j$ runs from $i+1$ to 6 . The transition-probability factor $w$, averaged over the angle $\chi$ of the perpendicular, detection radiofrequency field, is given by 9

$$
\begin{array}{r}
w=\left(\left|\left\langle i\left|\mathbf{e}_{\chi} \cdot \mathbf{S}\right| j\right\rangle\right|^{2}\right)_{\text {av over } x}={ }^{1} /{ }_{2}\left(\left|\left\langle i\left|S_{x}\right| j\right\rangle\right|^{2}+\left|\left\langle i\left|S_{y}\right| j\right\rangle\right|^{2}+\right. \\
\left.\left|\left\langle i\left|S_{z}\right| j\right\rangle\right|^{2}-\left|\left\langle i\left|\mathbf{e}_{0} \cdot \mathbf{S}\right| j\right\rangle\right|^{2}\right)
\end{array}
$$

where $e_{0}$ is a unit vector in the direction of $B$. The "effective $g$ factor", defined by

$$
g^{\prime}=h \nu / \beta B_{\text {res }}
$$

can be more informative than the value of the resonance field itself for those cases for which $g^{\prime}$ is less sensitive to the microwave frequency than $B_{\text {res. }}$.

To synthesize a magnetic resonance spectrum from the calculated resonance fields, one must put an appropriate lineshape function at each resonance field and then add together all the contributions. In the most general case, the spectrum from an individual molecule varies strongly with orientation; for randomly oriented molecules, such spectra have to be averaged over orientational angles. See ref 4 for more complete details of the spectral simulation.

Energy Levels vs Magnetic Field B. As an example, $\mathrm{Fe}^{3+}$ with spin of $5 / 2$ and with $D, E$, and $\nu$ as given above is considered. A typical plot of energy levels vs $B$ from 0 to $200 \mathrm{mT}$ is given in Figure 1. The orientation of $\mathbf{B}$ is $(\theta, \phi)=\left(65^{\circ}, 70^{\circ}\right)$. The four arrows in Figure 1 indicate the four instances at which the energy separations match the microwave frequency $\nu=9.22 \mathrm{GHz}$. The lowest Kramers doublet has a resonance at $97 \mathrm{mT}$; the highest, at $157 \mathrm{mT}$. The middle doublet has no resonance below $200 \mathrm{mT}$. Because $D$ and $E$ are small enough compared with $\nu$, there is also the possibility of a $2 \rightarrow 3$ interdoublet transition at $168 \mathrm{mT}$. The second and third energy levels have a minimum separation at about $184 \mathrm{mT}$, and as they separate beyond that, there is a second interdoublet resonance at $199 \mathrm{mT}$. These energy levels are highly anisotropic. For instance, if $\theta>70^{\circ}$, no interdoublet 2-3 resonance is possible at $\nu=9.22 \mathrm{GHz}$.

\section{Density Dot Plots}

Since the resonance fields vary with angle, the spectral contributions from molecules with random orientations tend to cancel out, except for those orientations at which the resonance fields are stationary - the turning points-and which dominate

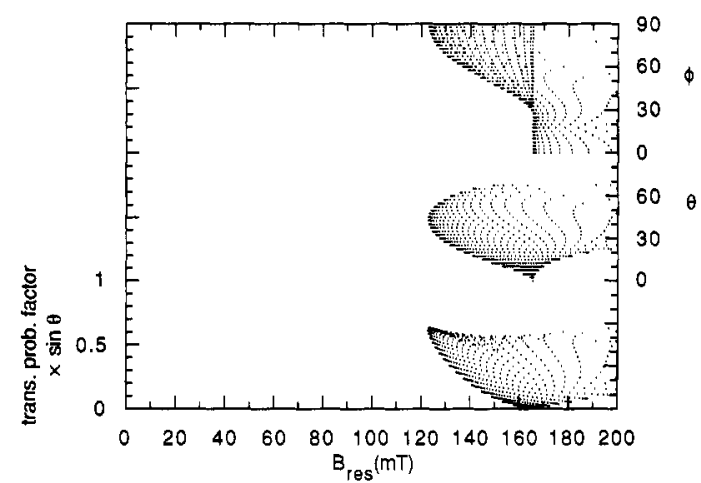

Figure 2. Density dot plot for the $2 \rightarrow 3$ interdoublet transition for $S$ $=s / 2 \mathrm{Fe}^{3+}$ with $D=0.26 \mathrm{~cm}^{-1}, E=0.026 \mathrm{~cm}^{-1}$, and $\nu=9.22 \mathrm{GHz}$. The angles $\theta$ and $\phi$ were varied from 0 to $90^{\circ}$ in 2.5-deg increments. For each resonance field found, there is a $\operatorname{dot}$ for $\theta$, for $\phi$, and for the $(\sin \theta)$ weighted transition-probability factor.

the spectrum. The natural question that arises is what molecules are contributing to what features of the spectrum. One graphical answer can be provided easily from a data file that contains a list of the resonant fields and transition-probability factors as a function of the angles. We call this a density dot plot, in which we plot three dots for each calculation, with $B_{\text {res }}$ along the horizontal axis, and with $\theta, \phi$, and $w \sin \theta$ along the vertical axis. [ $w$ is the transition-probability factor, eq 3.] Figure 2 is a density dot plot for the $2 \rightarrow 3$ transition described above, with $\theta$ and $\phi$ running from 0 to $90^{\circ}$ in 2.5 -deg increments. One can see immediately from the pile-up of dots near $B=123 \mathrm{mT}$ that there is an off-axis turning point in the $y z$ plane $\left(\phi=90^{\circ}\right)$ at $\theta \sim 43^{\circ}$ and that there is significant intensity. The spectral feature near $B=123 \mathrm{mT}$ reported below will thus be understood as having this origin. One can also see that there are no resonances with $\theta>70^{\circ}$. What is not easy to see from the density dot plot is, given the values of $\theta$ and $\phi$, at what field does the resonance occur.

One can generate a density dot plot by reading a list of $(\theta, \phi$, $B_{\text {res }}, w$ ) into a plotting program, such as KaleidaGraph, creating a plot with the $B_{\text {res }}$ column chosen for the horizontal axis, with all points shown, and with no lines drawn between points. Since no lines are drawn, the $B_{\text {res }}$ values do not have to appear in increasing order. (The ordering our program produces is indexed by $\theta$ and $\phi$, which is the order in which they are calculated.)

\section{Spherical Colored-Dot Plots}

The second graphical approach we present displays resonance information vs $\theta$ and $\phi$. The basic idea is to start with a portion of a sphere-a quadrant-with a grid to be able to identify values for $\theta$ and $\phi$. Then a dot is put at the end of a ray at a radius that indicates the value of $B_{\text {res. }}$. The dot is given a color to indicate the value of the transition-probability factor $w$. The same table of information used for the density dot plot is the raw material for the spherical colored-dot plot, but the information is presented more cleanly. Figure 3 is an example of the same $2 \rightarrow 3$ transition as for Figure 2, except that the values of $\theta$ and $\phi$ increase by 5 -deg increments. The color scheme for $w$ is that violet is $w=0$, with blue, green, and yellow being passed through in sequence as $w$ increases to its maximum value.

Parts c and d of Figure 3 give two views of $B_{\text {res, }}$, while parts $a$ and $b$ of Figure 3 give two views of the effective $g$ factor. A plot of $g^{\prime}$ gives the same information as a plot of $B_{\text {res, }}$, but some aspects of the visualization are easier to grasp. In particular, we point out the following: (1) There is a large region of the quadrant in which there are no reasonance fields less than $200 \mathrm{mT}$. (2) The transition-probability factor vanishes in the $z$ direction. (3) The change in $g^{\prime}\left(B_{\text {res }}\right)$ is greatest at the edges and has a maximum at $\theta \sim 45^{\circ}$ in the $y z$ plane. (4) At $\theta=65^{\circ}$ and $\phi=70^{\circ}$ and $110^{\circ}$, there are two dots on each ray. The second dot is the second 2 


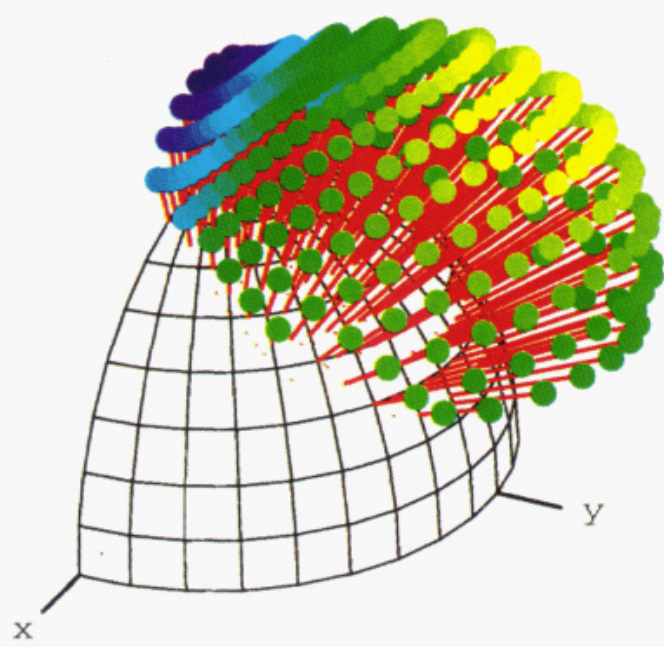

(a)

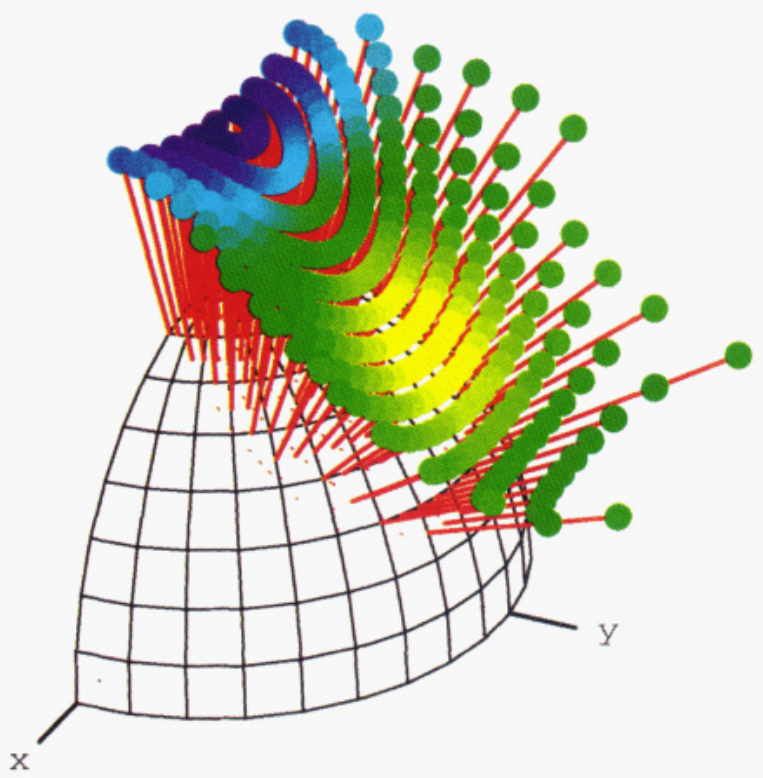

(c)
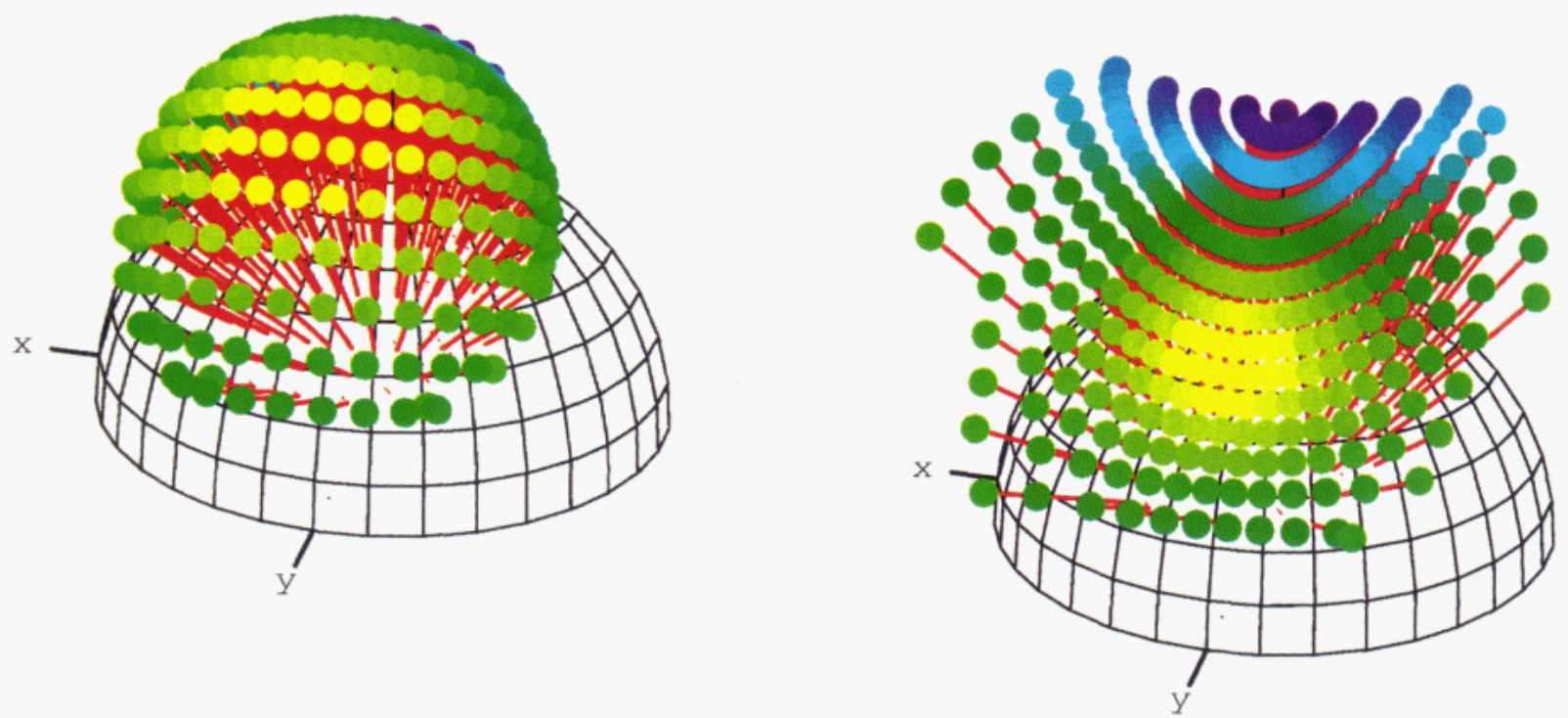

(b)

(d)

Figure 3. Spherical colored-dot plot for the $2 \rightarrow 3$ interdoublet transition for $S=5 / 2 \mathrm{Fe}^{3+}$ with $D=0.26 \mathrm{~cm}^{-1}, E=0.026 \mathrm{~cm}^{-1}$, and $\nu=9.22 \mathrm{GHz}$. The angles $\theta$ and $\phi$ were varied from 0 to $90^{\circ}$ and from 0 to $180^{\circ}$, respectively, both in 5-deg increments. For each resonance field found, there is a dot along the ray $(\theta, \phi)$. The color of the dot indicates the transition-probability factor: (violet $\rightarrow$ yellow) $\leftrightarrow(0 \rightarrow$ maximum). The radius of the ray is the magnitude of $g^{\prime}$ in a and b and the magnitude of $B_{\text {res }}$ in $\mathrm{c}$ and d.

$\rightarrow 3$ transition near $199 \mathrm{mT}$ shown in Figure 1 . This is the only direction shown in Figure 3 at which there are two resonance fields. (5) In fact, the $2 \rightarrow 3$ transition is between two levels that at zero field are far apart, that (at any angle) approach each other, avoid crossing, and then again separate. If the two levels come close enough to permit a transition, then there will be a second transition on the other side of the minimum separation, at a higher field (unless the transition occurs exactly at the minimum). Thus, there will always be a companion second dot at higher field, and the surface traced out by the dots will tend to close up. The 200-mT maximum in the plot was picked because it included the part of the experiment that was most relevant, but it also had the consequence of keeping Figure 3 easy to visualize. (6) The reflection symmetry between the first octant and the second $\left(90^{\circ}<\phi<180^{\circ}\right)$ is apparent.

One can generate a spherical colored-dot plot via Mathematica. The code to produce such a plot with data read from a file called DataFile, each line of which contains the information $\left(B_{\mathrm{res}}, \theta, \phi\right.$, $w)$, is given in Chart I.

\section{Application: The Oxalate Complex of Diferric Transferrin}

Choice of Zero-Field Parameters $D$ and $E$ for the Experimental Spectra of Transferrin Oxalate. The $D$ value for high-spin ferric iron in the carbonate complex of transferrin is well established 


\section{CHART I}

eprData = ReadList ["DataF1le",Number, RecordLists->True]; bmin =Min [Table [eprData [ [n] $[[1]],\{n, 1$, Length [eprData] $]]$; tpmax $=\operatorname{Max}[$ Table $[$ eprData $[[n]][\{4]\},\{n, 1$, Length [eprData $]\}]\}$; tpmin=Min [Table [eprData $[[n]][[4]\},\{n, 1$, Length [eprData])]]; $\operatorname{cf}\left[x_{-}\right]:=0.15+0.55{ }^{*} x /(t p \operatorname{pmax}-t p m 1 n) ;$

$x\left[t_{-}, P_{-}\right]:=N[\sin [t] \cos [P]] ; y\left[t, P_{-}\right]:=N[\sin [t] \sin [p]] ;$ $z\left[t_{-}\right]:=N[\cos [t]] ;$

radialbData = Flatten[\{Po1ntSize[0.03],

eprData/.\{b_Real, t_Real,p_Real,tp_Real $\} \rightarrow$ [

Hue $[c f[t p]]$, PoInt $\left[\left\{b^{\star} x[t, p], b^{*} y[t, p], b^{\star} z[t]\right\}\right]$

\}, Hue [0.0],

eprData/. \{b_Real,t_Real,p_Real,tp_Real\} $\rightarrow$ |

Li ne $\left.\left.\left[\left\{(0,0,0\},.\left\{b^{\star} x[t, p], b^{\star} y[t, p], b^{\star} z[t]\right\}\right\}\right]\right\}, 2\right]$;

bplot = Graphics3D [ radialbData,

ViewPolnt->\{2.769, 1.189, 1.540\}, Boxed->Faise,

Lighting->False, D1splayFunction->Ident1ty];

sframe $=$ Paramet $r 1 c 81$ ot $3 D[\{.9 \star b m 1 n \star x[t, p], .9 \star b m 1 n \star y[t, p]$,

$.9 * b m i n * z[t]\},\{t, 0, P i / 2\},\{p, 0, P 1\}$,

ViewPoint-> $12.769,1.189,1.540\}$, Shading->False,

Boxed->False, Ilghting->False, Axes->False,

Plotpoints $\rightarrow\{10,19\}$, DisplayFunction->Ident1ty];

$x y z=$ Graph1es3D [

$\{$ Iine $[\{10,0,0\},\{1.1 *$ bmin, 0,0$\}\}]$, Text $\{" x ",\{1.2 * b m i n, 0,0\}\}$,

Line $[\{10,0,0\},(0,1.1 * b m i n, 0)\}], T e x t[" y ", 10,1.2 \star b m i n, 0\}]$, Line $[\{(0,0,0\},\{0,0,1.1 * b m i n\}\}\}]$;

Show [ \{xyz, sframe, bplot\}, V1ewPolnt-> $\{2.769,1.189,1.540\}$, Shadlng->False, Boxed->False, Lighting->False, Axes->False, DisplayFunct 1 on->\$D1splayFunction]

at about $0.25 \mathrm{~cm}^{-1}$, from EPR and Mössbauer measurements. ${ }^{7,10}$ The symmetry of the iron center in transferrin changes as different bidentate anions are substituted for carbonate. ${ }^{7,8,11}$ The $\mathrm{X}$-band EPR spectra of many of these complexes show two broad maxima in the region from 40 to $200 \mathrm{mT}$. These features result from contributions from transitions between more than one pair of energy levels. Initial fits of simulated and experimental spectra emphasized contributions from two Kramers doublets to this spectral region. ${ }^{8}$ In this spectra, we examine the spectra of the oxalate complex of transferrin in more detail and address whether accurate values of $D$ and $E$ for the oxalate complex can be obtained from the EPR spectra alone.

The lowest field peak in the experimental X-band EPR spectrum of transferrin oxalate in low salt buffer is at $86 \pm 2 \mathrm{mT}$. As an aid in assigning this, and other features in the spectrum, Figure 4 shows a summary of the fields at which maxima in calculated spectra occur as a function of $E / D$. As shown above, when $D$ $\sim h \nu$, regions of high intensity in the spectrum do not necessarily coincide with principal axis directions, so Figure 4 was generated from simulated powder patterns in derivative form. The dependence of resonance fields on the value of $D$ is also given in separate panels in this figure. From the figure, if $D>0.25$, the maximum of the low field feature of the transferrin oxalate spectrum must arise from a transition between levels 1 and 2 . The value of $E / D$ that gives a maximum at $86 \mathrm{mT}$ is $\sim 0.095$ for any $D>0.25$. This choice of $E / D$ places a second maximum in the spectrum from the 3 to 4 transition in the range from 115 to 120 $\mathrm{mT}$. The experimental value is in this range for transferrin oxalate in low salt buffer. A fine tuning of the comparison of experimental and simulated spectra leads to $E / D=0.095$ and $D=0.260 \mathrm{~cm}^{-1}$ for the low salt, transferrin oxalate sample. A number of biochemical variables, in addition to bidentate ligands, affect EPR spectra of transferrin complexes. Addition of high concentrations of sodium chloride is one of these variables. 8,11 Addition of salt to $1.0 \mathrm{M}$ to the sample discussed above changes the apparent maxima in the spectra to 90.6 and $113 \mathrm{mT}$. In reference again to Figure 4, parameters that result in the observed features for the high salt sample are approximately $E / D=0.07$ and $D=0.262 \mathrm{~cm}^{-1}$. The number next to plots in Figure 4 are transition-probability factors. These indicate that transitions between levels 1 and 2 and between levels 3 and 4 should be of similar magnitude for the range of $E / D$ from 0.06 to 0.10 . This is the range of $E / D$ values for transferrin oxalate.

LineShapes. Aasa and Vänngard ${ }^{12}$ pointed out that the formula for the absorption of radiation from time-dependent perturbation theory pertains to "frequency-swept" spectra and is normalized so that the integral of the line-shape function over frequency, not over field, is unity,

$$
\int_{-\infty}^{\infty} \frac{f_{\text {freq }}\left(\left|\nu-\nu_{\text {res }}\right| / \sigma_{\text {freq }}\right)}{\sigma_{\text {freq }}} \mathrm{d} \nu=1
$$

In the usual experiment the field is swept: $\nu$ is held fixed at $\nu_{0}$, and $\nu_{\text {res }}$ is varied by sweeping $B$. The line-shape function, $f_{\text {freq }}\left(\mid \nu_{0}\right.$ $\left.-\nu_{\text {res }} \mid / \sigma_{\text {freq }}\right) / \sigma_{\text {freq }}$, can be expressed in terms of magnetic field $(B)$ via the following:

$$
\begin{gathered}
\text { resonance field } B_{0}: \quad E_{\text {upper }}\left(B_{0}\right)-E_{\text {lower }}\left(B_{0}\right)=h \nu_{0} \\
\nu_{\text {res }} \equiv\left[E_{\text {upper }}(B)-E_{\text {lower }}(B)\right] / h \\
=\nu_{0}+\left(B-B_{0}\right) \frac{\mathrm{d}\left[E_{\text {upper }}\left(B_{0}\right)-E_{\text {lower }}\left(B_{0}\right)\right]}{\mathrm{d} B_{0}} / h+1 \ldots \\
\frac{f_{\text {freq }}\left(\left|\nu_{0}-\nu_{\text {res }}\right| / \sigma_{\text {freq }}\right)}{\sigma_{\text {freq }}}= \\
\frac{1}{\sigma_{\text {freq }}} f_{\text {freq }}\left(\frac{\left|B-B_{0}\right|}{h \sigma_{\text {freq }}} \frac{\mathrm{d}\left[E_{\text {upper }}\left(B_{0}\right)-E_{\text {lower }}\left(B_{0}\right)\right]}{\mathrm{d} B_{0}}+\ldots\right) \\
=\frac{1}{\sigma_{\text {freq }}} f_{\text {freq }}\left(\left|B-B_{0}\right| / \sigma_{\text {field }}\right)+\ldots
\end{gathered}
$$

Consequently, the appropriate width $\sigma_{\text {field }}$ to use for fieldswept spectra is obtained from the frequency-swept width $\sigma_{\text {freq }}$ via

$$
\sigma_{\text {field }}=\sigma_{\text {freq }} \frac{h}{\mathrm{~d}\left[E_{\text {upper }}\left(B_{0}\right)-E_{\text {lower }}\left(B_{0}\right)\right] / \mathrm{d} B_{0}}
$$

Equation 11 is the field-variable line-width term used for the calculations presented here.

Comparison of eqs 5 and 10 reveals that the line-shape function derived for the field-swept spectrum (to leading order in $B-B_{0}$ ) has the same functional form as for the frequency-swept spectrum, but that the right-hand side of eq 8 will not integrate to unity with respect to $B$. The integrated intensity with respect to $B$ is instead $\sigma_{\text {field }} / \sigma_{\text {freq }}$,

$$
\frac{\sigma_{\text {field }}}{\sigma_{\text {freq }}}=\frac{h}{\mathrm{~d}\left[E_{\text {upper }}\left(B_{0}\right)-E_{\text {lower }}\left(B_{0}\right)\right] / \mathrm{d} B_{0}}
$$

In the low field case, the energy levels are linear functions of $B$, and eq 11 simplifies to the Aasa-Vänngård " $1 / g^{\prime}$ factor":

$$
\frac{\sigma_{\text {field }}}{\sigma_{\text {freq }}} \sim \frac{B_{0}}{\nu_{0}}=\frac{h}{g^{\prime} \beta}
$$

An example of the effect of the line-shape function on the outcome of a spectral simulation is given in Figure 5. Here, the low field region is shown separately for transitions between levels 1 and 2, 2 and 3, and 3 and 4. The parameters chosen for the calculation are in the range appropriate for simulation of transferrin oxalate spectra. The calculation for the spectrum shown by a solid line used the derivative line-shape function of eq 11 , while the calculation giving the dashed line used the lineshape function that assumes linear dependence between resonant field and frequency, eq 13. Clearly the 2-3 transition depends more strongly on the line-shape function than do the 1-2 and 3-4 transitions.

Fit to Experimental Spectrum. The next two considerations in comparing experimental and simulated spectra are whether 

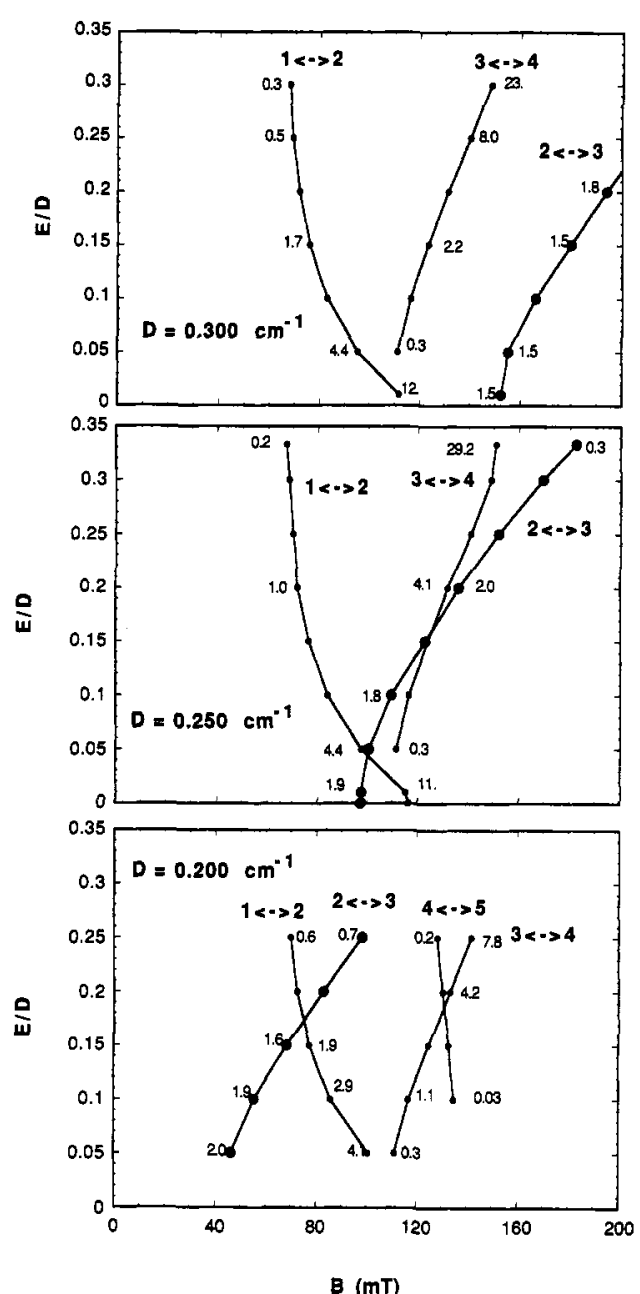

Figure 4. Plots of the variation of the fields at which the calculated $\mathrm{X}$-band EPR powder derivative spectra have maxima, as a function of $E / D$. Calculations for each pair of energy levels were analyzed separately. Only the position of the lowest field feature, for transitions in a particular pair of levels, is shown. The variation of these plots with the value of $D$ is also shown. The magnitude of $D$ decreases from $0.3 \mathrm{~cm}^{-1}$ at the top to $0.2 \mathrm{~cm}^{-1}$ at the bottom. For this range of $D$, transitions between three pairs of energy levels (1-2,2-3, and 3-4) should contribute significantly to the observed spectra in the range from 0 to $200 \mathrm{mT}$. At the lowest value of $D$ shown, $0.2 \mathrm{~cm}^{-1}$, transitions between levels 4 and 5 may also contribute to this region of the spectra. Numbers given at selected points on the plots are relative transition probabilities. The data for transitions between levels 2 and 3 are shown with enlarged circles to emphasize how strongly the resonant field for these transitions depends on the value of $D$.

there is evidence for distributions in the values of $D$ or $E$, as there are for the carbonate complex of transferrin (10), and whether large line widths or distributions in other parameters shift the positions of apparent maxima in the spectra. The shape of the lowest field maximum in the experimental spectrum for transferrin oxalate in low salt is asymmetric, being broader on the high field side. The opposite asymmetry, i.e. broader to low field, is characteristic of the low field feature for samples in high salt. The data in Figure 4 show that asymmetric broadening to the high field side is expected if a distribution in values of $E / D$ characterizes the spectra. Starting with the values of $E / D$ deduced above, variations in line width and distributions in $E / D$, with $D$ held constant, have been applied to fit the experimental spectra of transferrin oxalate in low and high salt buffers. The result is shown in Figure 6, which gives two simulations (solid lines) superimposed on experimental spectra (noisy lines). The upper set of spectra are for transferrin oxalate in low salt buffer, and the lower ones are for the sample in $0.1 \mathrm{M}$ salt. Using a line width of $350 \mathrm{MHz}$, the low field feature (1-2 transition) of the upper spectrum was fit better with an $E / D$ distribution than

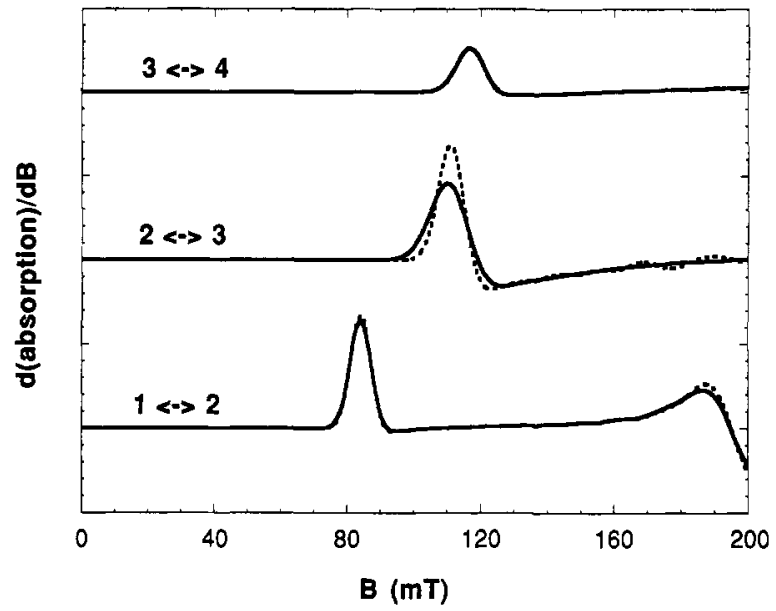

Figure 5. Low field region of calculated, derivative powder pattern spectra, showing the effect of the line-shape calculation on the shape of the low field feature. Transitions between levels 1 and 2,2 and 3, and 3 and 4 are shown separately. The derivative line-shape function, eq 11 , was used for the spectrum shown by a solid line; eq 13 was used for the calculation giving the dashed line. Parameters for this calculation were $D=0.25 \mathrm{~cm}^{-1}, E / D=0.1$, and frequency-swept line width $=150 \mathrm{MHz}$.

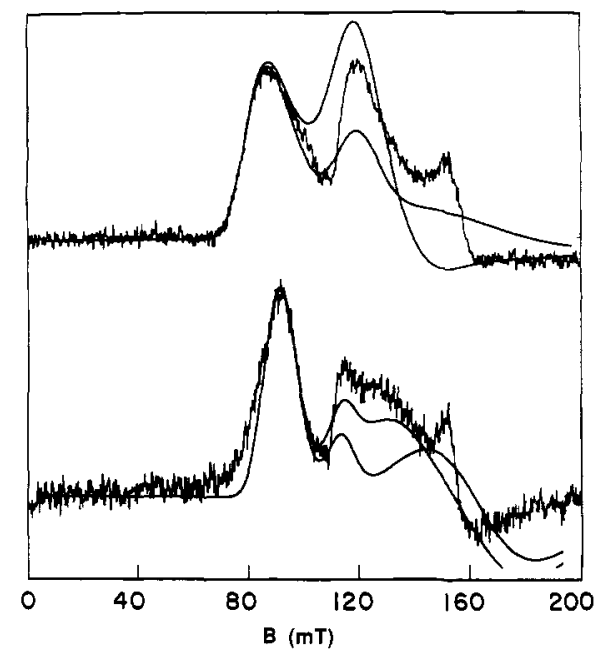

Figure 6. Experimental X-band EPR spectra of transferrin oxalate at $4 \mathrm{~K}$ (noisy lines) compared with simulations (smooth lines). The simulated spectra show two calculations. In one case, the simulation is the sum of transitions between Kramers doublets (levels 1 and 2 and levels 3 and 4). In the other case, giving higher intensity near $120 \mathrm{mT}$, interdoublet transitions between levels 2 and 3 are included with the other two. The upper set of spectra are for transferrin oxalate in buffer composed of 0.09 $M$ HEPES, $35 \mathrm{mM}$ oxalic acid, and ammonium hydroxide to adjust the $\mathrm{pH}$ to 7.5. The lower set are for the same sample diluted $1: 1$ with $2 \mathrm{M}$ sodium chloride. The simulation parameters are, for the upper spectra, $E / D=0.095, D=0.26 \mathrm{~cm}^{-1}$, width of distribution in $E / D=0.20$, and line width $=350 \mathrm{MHz}$ [using line width formula 11]. For the lower spectra, the simulation parameters are $E / D=0.070, D=0.262 \mathrm{~cm}^{-1}$, width of distribution in $E / D=0.17$, and line width $=350 \mathrm{MHz}$ [again, using line-width formula 11]. (The experimental spectra are reproduced with permission from Figure 5 of ref 8 .)

without. A Gaussian distribution in $E / D$ of half-width 0.02 gave the best fit, and that simulation is given in the figure. (Other line widths and $E / D$ distributions gave poorer fits.) Two simulations are shown: the one with lower intensity at $120 \mathrm{mT}$ has contributions only from the $1-2$ and 3-4 transitions. The one with higher intensity at $120 \mathrm{mT}$ includes the calculated contribution from the 2-3 transition. The lower portion of Figure 6 shows the fits for the high salt sample. The simulations differ in $E / D$, as noted above, and the distribution in $E / D$ was slightly smaller for the lower figure (half-width in $E / D=0.017$ ). Again, the intensity is calculated to be higher near $120 \mathrm{mT}$ if a contribution from the 2-3 transition is included. 
Further assignment of the parameters that characterize the $X$-band EPR spectra of transferrin oxalate does not seem possible with the limited experimental data in Figure 6. Although some mixture of the upper and lower simulations might improve the result for each case, ${ }^{8}$ it is clear that the second feature at $\sim 120$ $\mathrm{mT}$ is more intense in the experimental spectra than in calculations that omit a contribution from the 2-3 transition. Because the resonance position of the 2-3 transition is so sensitive to the value of $D$ (see Figure 4), a small distribution in $D$ would broaden the feature from the 2-3 transition more than the other features in the spectrum. Thus, one set of solutions to fitting the experimental spectra would employ a value of $D$ close to $0.26 \mathrm{~cm}^{1}$ and a distribution in both $D$ and $E$. Other approaches to giving intensity in this region would be addition of another spectrum for a species with axial symmetry or choosing a distribution different from the gaussian $E / D$ distribution we have used. To resolve these possibilities, EPR spectra at slightly different magnetic fields might be examined, or data to further define $D$ and $E$ could be obtained by optical or Mössbauer methods.

Acknowledgment. The authors dedicate this paper to Harden M. McConnell and thank him for encouragement over many years. We gratefully acknowledge the support of The National Institutes of Health, Grant No. R01 GM36232.

\section{References and Notes}

(1) Bennett, J. E.; Gibson, J. F,; Ingram, D. J. E. Proc. R. Soc. London A $1957,240,67$.

(2) Coffman, R. E. J. Phys. Chem. 1975, 79, 1129.

(3) Feher, G, Electron Paramagnetic Resonance with Applications to Selected Problems in Biology (Les Houches Lectures, 1969); Gordon and Breach: New York, 1970.

(4) Gaffney, B. J.; Silverstone, H. J. Simulation of the EMR Spectra of High Spin Iron in Proteins. In Biological Magnetic Resonance, EMR of Paramagnetic Molecules; Berliner, L. J., Reuben, J., Eds.; Plenum Press: New York, 1993.

(5) Zhang, Y.; Gebbhard, M. S.; Solomon, E. I. J. Am. Chem. Soc. 1991, 113,5162 .

(6) Howard, J. B.; Rees, D. C. Perspectives on Non-Heme Iron Protein Chemistry. Advances in Protein Chemistry; Academic Press: New York, 1991; Vol. 42, p 199.

(7) Aasa, R. J. Chem. Phys. 1970, 52, 3919.

(8) Dubach, J.; Gaffney, B. J.; More, K.; Eaton, G. R.; Eaton, S. S. Biophys. J. 1991, 59, 1091 .

(9) The right-hand side of eq 3 is obtained in two steps. Direct averaging over $\chi$, the angular coordinate in the plane perpendicular to $e_{0}$, the direction of $\mathbf{B}_{0}$, gives $w=1 / 2\left(\left|\left\langle i\left|\cos \theta \cos \phi S_{x}+\cos \theta \sin \phi S_{y}-\sin \theta S_{z}\right| j\right\rangle\right|^{2}+\mid\langle i|-\sin \right.$ $\left.\phi S_{x}+\left.\cos \phi S_{y}|j\rangle\right|^{2}\right)$. Then expansion of the squares, combined with trigonometric identities, finishes the task.

(10) Kretchmar, S. A.; Teixeira, M.; Huynh, B.-H.; Raymond, K. N. Biol. Met. 1988, 1, 26.

(11) Yang, A.-S.; Gaffney, B. J. Biophys. J. 1987, 51, 55.

(12) Aasa, R.; Vänngåd, T. J. Magn. Res. 1975, 19, 308. 